\title{
Optimal Stable Solution of Cauchy Problems for Elliptic Equations
}

\author{
U. Tautenhahn
}

\begin{abstract}
We consider ill-posed Cauchy problems for elliptic partial differential equations $u_{u}-L u=0\left(0<t \leq T, x \in \Omega \subset \mathbb{R}^{n}\right)$ with linear densely defined self-adjoint and positive definite operators $L: D(L) \subset H \rightarrow H$ where $H$ denotes a Hilbert space with norm $\|\cdot\|$ and inner product $(\cdot, \cdot)$. We assume that instead of exact data $y=u(x, 0)$ or $y=u_{t}(x, 0)$ noisy data $y^{\delta}=u^{\delta}(x, 0)$ or $y^{\delta}=u_{t}^{\delta}(x, 0)$ are available, respectively, with $\left\|y-y^{\delta}\right\| \leq \delta$. Furthermore we assume certain smoothness conditions $u(x, t) \in M$ with appropriate sets $M$ and answer the question concerning the best possible accuracy for identifying $u(x, t)$ from the noisy data. For special sets $M$ the best possible accuracy depends either in a Hölder continuous way or in a logarithmic way on the noise level $\delta$. Furthermore, we discuss special regularization methods which realize this best possible accuracy.
\end{abstract}

Keywords: Ill-posed problems, elliptic partial differential equations, Cauchy problems, optimal regularization methods, optimal error bounds

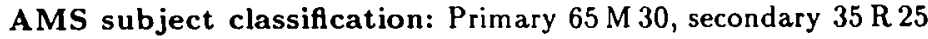

\section{Introduction}

We consider the elliptic equation

$$
u_{t t}-L u=0 \quad \text { for } 0<t \leq T \text { and } x \in \Omega \subset \mathbb{R}^{n}
$$

where $L: D(L) \subset H \rightarrow H$ denotes a linear densely defined self-adjoint and positive definite operator with eigenvalues $l_{i}(i \geq 1)$ such that

$$
0<l_{1} \leq l_{2} \leq \ldots \quad \text { and } \quad l_{i} \rightarrow \infty \text { for } i \rightarrow \infty
$$

and eigenelements $u_{i}$ that form an orthonormal basis in a real Hilbert space $H$ with norm $\|\cdot\|$ and inner product $(\cdot, \cdot)$. A first example for $(1.1)$ is the Laplace equation in two dimensions

$$
\left.\begin{array}{ll}
u_{t t}+u_{x x}=0 & \text { for } t \in(0, T) \text { and } x \in(0, \pi) \\
=u(\pi, t)=0 & \text { for } t \in[0, T]
\end{array}\right\}
$$

U. Tautenhahn: Hochschule für Technik, Wirtschaft und Sozialwesen (FH) Zittau/Görlitz, FB Math./Naturwiss., PSF 261, D - 02755 Zittau 
in which the eigenvalues $l_{i}$ and eigenelements $u_{i}$ of $L: H_{0}^{1}(0, \pi) \cap H^{2}(0, \pi) \subset H \rightarrow H$ with $H=L^{2}(0, \pi)$ are given by

$$
l_{i}=i^{2} \quad \text { and } \quad u_{i}=\sqrt{\frac{2}{\pi}} \sin (i x)
$$

$(i \geq 1)$. A second example for $(1.1)$ is the Laplace equation in three dimensions

$$
\left.\begin{array}{rlrl}
u_{t t}+u_{x_{1} x_{1}}+u_{x_{2} x_{2}}=0 & & \text { for } t \in(0, T) \text { and } x \in \Omega=(0, c) \times(0, d) \\
u(x, t)=0 & \text { for } t \in[0, T] \text { and } x \in \partial \Omega
\end{array}\right\}
$$

in which the eigenvalues $l_{i j}$ and eigenelements $u_{i j}$ of $L: H_{0}^{1}(\Omega) \cap H^{2}(\Omega) \subset H \rightarrow H$ with $H=L^{2}(\Omega)$ are given by

$$
l_{i j}=\left(\frac{i \pi}{c}\right)^{2}+\left(\frac{j \pi}{d}\right)^{2} \quad \text { and } \quad u_{i j}=\frac{2}{\sqrt{c d}} \sin \frac{i \pi x_{1}}{c} \sin \frac{j \pi x_{2}}{d}
$$

$(i, j \geq 1)$ (see, e.g., [4]). Connected with equation (1.1) we formulate the two following problems.

Problem (P1) (Identification of $u(x, t)$ from $u(x, 0)$ ). Given $u_{t}(x, 0)=0$ and noisy data $u^{\delta}(x, 0) \in H=L^{2}(\Omega)$ to $u(x, 0)$, find (for some fixed $\left.t \in(0, T]\right)$ the solution $u(x, t)$ of problem (1.1) for $x \in \Omega$.

Problem (P2) (Identification of $u(x, t)$ from $u_{t}(x, 0)$ ). Given $u(x, 0)=0$ and noisy data $u_{t}^{\delta}(x, 0) \in H=L^{2}(\Omega)$ to $u_{t}(x, 0)$, find (for some fixed $t \in(0, T]$ ) the solution $u(x, t)$ of problem (1.1) for $x \in \Omega$.

Problems (P1) and (P2) for the Laplace equation (1.2) are the classical ill-posed problems of Hadamard (cf. [5]) for which there do not exist any solutions in general.

If for problem (P1) the noisy data are given by $u^{\delta}(x, 0)=\frac{\sin k x}{k^{3}}$, then we have a solution $u^{\delta}(x, t)=\frac{\sin k x \cosh k t}{k^{3}}$, however though $u^{\delta}(x, 0)$ and its first and second derivative tend to zero for $k \rightarrow \infty$, the corresponding solutions $u^{\delta}(x, t)$ tend to infinity for any $t \in(0, T]$ and $x \in(0, \pi)$.

If for problem (P2) the noisy data are given by $u_{t}^{\delta}(x, 0)=\frac{\sin k x}{k^{3}}$, then we have a solution $u^{\delta}(x, t)=\frac{\sin k x \sinh k t}{k^{4}}$, however though $u_{t}^{\delta}(x, 0)$ and its first and second derivative tend to zero for $k \rightarrow \infty$ the corresponding solutions $u^{\delta}(x, t)$ tend to infinity for any $t \in(0, T]$ and $x \in(0, \pi)$.

Our formulated problems (P1) and (P2) are ill-posed: their solutions (if they exist) do not depend continuously on the data. Since the data $y=u(x, 0)$ for problem $(\mathrm{P} 1)$ and $y=u_{t}(x, 0)$ for problem (P2), respectively, are based on (physical) observations and not known with complete accuracy (the known noisy data are $y^{\delta}=u^{\delta}(x, 0)$ for problem (P1) and $y^{\delta}=u_{t}^{\delta}(x, 0)$ for problem (P2), respectively), for a stable numerical approximation of the solution $u=u(x, t)$ of problems (P1) and (P2) some regularization technique has to be applied, which provides a sequence of approximations $u_{\alpha}^{\delta}(x, t)=R_{\alpha}(t) y^{\delta}$ with property $u_{\alpha}^{\delta}(x, t) \rightarrow u(x, t)$ as $\delta:=\left\|y-y^{\delta}\right\| \rightarrow 0$ where the regularization parameter 
$\alpha=\alpha(\delta)$ has to be chosen properly. Hence, regularized solutions $u_{\alpha}^{\delta}=u_{\alpha}^{\delta}(x, t)$ depend continuously on the data. However, the convergence of $u_{\alpha}^{\delta}$ to $u$ can be arbitrarily slow without assuming additional quantitative a priori restrictions on the unknown solution $u$, which is typical for ill-posed problems. Quantitative a priori restrictions that will work in many ill-posed problems (and enable us to estimate the convergence rate) consist in imposing a bound $E$ on the (unknown) solution and a finite number of it's derivatives.

Let us describe our quantitative a priori information concerning $u(x, t)$ in more detail. We introduce a Hilbert scale $\left(H_{r}\right)_{r \in \mathbb{R}^{+}}$(cf. [10]) according to $H_{0}=H=L^{2}(\Omega)$ and $H_{r}=D\left(L^{\frac{r}{2}}\right)$ where

$$
\|u\|_{r}=\left\|L^{\frac{r}{2}} u\right\| \quad\left(r \in \mathbb{R}^{+}\right)
$$

is the norm in $H_{r}$ and require for both problems (P1) and (P2) the a priori smoothness condition concerning the unknown solution $u$ according to

$$
u(x, t) \in M_{p, E}=\left\{u(x, t) \in H \mid\|u(x, T)\|_{p} \leq E \text { for some } p \geq 0\right\} .
$$

For example, assumption (1.5) means for problem (1.3) that $\|u(x, T)\|_{L^{2}(\Omega)} \leq E$ in case $p=0$ or $\left\|u_{x_{1} x_{1}}(x, T)+u_{x_{2} x_{2}}(x, T)\right\|_{L^{2}(\Omega)} \leq E$ in case $p=2$. The larger $p$, the more restrictive is assumption (1.5).

Any operator $R(t): H \rightarrow H$ can be considered as a special method for identifying the solution $u(x, t)$ of problems (P1) or (P2) from noisy data $y^{\delta}=u^{\delta}(x, 0) \in H$ or $y^{\delta}=u_{t}^{\delta}(x, 0) \in H$, respectively; the approximate solution to problem (P1) or (P2) is then given by $R(t) y^{\delta}$. We introduce the worst case error $\Delta(\delta, R(t))$ for identifying $u(x, t)$ from $y^{\delta} \in H$ under the conditions $\left\|y-y^{\delta}\right\| \leq \delta$ and $u(x, t) \in M_{p, E}$ by

$$
\Delta(\delta, R(t))=\sup \left\{\left\|R(t) y^{\delta}-u(x, t)\right\| \mid u(x, t) \in M_{p, E}, y^{\delta} \in H,\left\|y-y^{\delta}\right\| \leq \delta\right\} .
$$

This worst case error characterizes the maximal error of an arbitrary method $R(t)$ if the, solution $u(x, t)$ varies in the set $M_{p, E}$ given in (1.5). Now we ask the question concerning the magnitude of the worst case error $\Delta(\delta, R(t))$ for 'optimal' methods $R(t): H \rightarrow H$ that minimize the worst case error (1.6) over all methods $R(t): H \rightarrow H$. In Section 2 we review a general formula for the best possible worst case error

$$
\omega(\delta, t)=\inf _{R(t)} \Delta(\delta, R(t))
$$

which shows us in which kind this best possible worst case error depends on the noise level $\delta$. In Sections 3 and 4 we apply this general optimality result to the above two problems (P1) and (P2). We prove that $\omega(\delta, t)=c_{1} \delta^{1-\frac{1}{T}}\left[\ln \frac{1}{\delta}\right]^{-p \frac{t}{T}}$ for problem (P1) and $\omega(\delta, t)=c_{2} \delta^{1-\frac{t}{T}}\left[\ln \frac{1}{\delta}\right]^{-p \frac{\dot{t}}{T^{2}}-\left(1-\frac{t}{T}\right)}$ for problem (P2) with certain constants $c_{1}$ and $c_{2}$. In Sections 5 and 6 we discuss special regularization methods for problems (P1) and (P2). We construct regularized approximations $u_{\alpha}^{\delta}(x, t)=R_{\alpha} y^{\delta}$ to their unknown solutions $u(x, t)$ (with $y^{\delta}=u^{\delta}(x, 0)$ or $y^{\delta}=u_{t}^{\delta}(x, 0)$, respectively) that guarantee 'optimal' error bounds $\left\|u_{\alpha}^{\delta}(x, t)-u(x, t)\right\| \leq \omega(\delta, t)$, hence, the constructed approximations $u_{\alpha}^{\delta}(x, t)$ are as accurate as possible in terms of the given information $\left\|y-y^{\delta}\right\| \leq \delta$ and (1.5). These constructed approximations require the knowledge of the smoothness parameter $p$, the a priori bound $E$ and the noise level $\delta$. 


\section{Optimal error bounds and regularization methods}

In this section we consider arbitrary ill-posed inverse problems

$$
A x=y
$$

where $A \in \mathcal{L}(X, Y)$ is a linear injective bounded operator between infinite-dimensional Hilbert spaces $X$ and $Y$ with non-closed range $R(A)$. Throughout this section we assume that $y^{\delta} \in Y$ are the available noisy data with $\left\|y-y^{\delta}\right\| \leq \delta$. Any operator $R: Y \rightarrow X$ can be considered as a special method for solving equation (2.1); the approximate solution to equation (2.1) is then given by $R y^{\delta}$.

Let $M \subset X$ a bounded set. We introduce the worst case error $\Delta(\delta, R)$ for identifying $x$ from $y^{\delta} \in Y$ under the assumptions $\left\|y-y^{\delta}\right\| \leq \delta$ and $x \in M$ according to

$$
\Delta(\delta, R)=\sup \left\{\left\|R y^{\delta}-x\right\| \mid x \in M, y^{\delta} \in Y,\left\|A x-y^{\delta}\right\| \leq \delta\right\} .
$$

This worst case error characterizes the maximal error of the method $R$ if the solution $x$ of problem (2.1) varies in the set $M$. Parameter dependent methods $R=R_{\delta}$ are called

(i) optimal on the set $M$ if $\Delta\left(\delta, R_{\delta}\right)=\inf _{R: Y \rightarrow X} \Delta(\delta, R)$

(ii) order optimal on the set $M$ if $\Delta\left(\delta, R_{\delta}\right) \leq \operatorname{cinf}_{R} \Delta(\delta, R)$ with $c \geq 1$.

For a general discussion of optimality of parameter-dependent regularization methods $R_{\delta}$ in the special case that the set $M$ is given by

$$
M=\left\{x \in X \mid x \stackrel{*}{=}\left(A^{*} A\right)^{\frac{2}{2}} v,\|v\| \leq E, p>0\right\} .
$$

we refer to $[12,14,16,19]$; concerning order optimality we refer to $[1,2,12,20]$.

In this section we review some optimality. results if the set $M$ is given by

$$
M_{\varphi, E}=\left\{x \in X \mid x=\left[\varphi\left(A^{*} A\right)\right]^{\frac{1}{2}} v,\|v\| \leq E\right\}
$$

where the operator function $\varphi\left(A^{*} A\right)$ is well defined via spectral representation $\varphi\left(A^{*} A\right)=$ $\int_{0}^{a} \varphi(\lambda) d E_{\lambda}$ where $A^{*} A=\int_{0}^{a} \lambda d E_{\lambda}$ is the spectral decomposition of $A^{*} A,\left\{E_{\lambda}\right\}$ denotes the spectral family of the operator $A^{*} A$ and $a$ is a constant with $\left\|A^{*} A\right\| \leq a$. In the case of compact operators $A \in \mathcal{L}(X, Y)$ the operator function $\varphi\left(A^{*} A\right)$ attains the form

$$
\varphi\left(A^{*} A\right) x=\sum_{i=1}^{\infty} \varphi\left(\lambda_{i}\right)\left(x, u_{i}\right) u_{i}
$$

for all $x \in X$, where $\lambda_{i}(i \geq 1)$ are the (positive) eigenvalues with $\lambda_{1} \leq \lambda_{2} \leq \ldots$. and $\lambda_{i} \rightarrow 0$ for $i \rightarrow \infty$, and $u_{i}$ are the eigenelements of the compact operator $A^{*} A \in$ $\mathcal{L}(X, X)$.

Let us motivate the practical relevance of restricting attention to the general source set (2.3). In different ill-posed differential equation problems (2.1) additional quantitative a priori restriction is given by a certain smoothness of the unknown solution $x \in X$. 
Such smoothness conditions can be reformulated into equivalent conditions $x \in M_{\varphi, E}$ with certain functions $\varphi=\varphi(\lambda)$ which generally are not of the form $\varphi(\lambda)=\lambda^{p}$. Hence, the set $M_{\varphi, E}$ does not coincide with the above set $M$ in general, and the general optimality results known for the above set $M$ cannot be applied. Let us discuss this fact for the heat equation problem backward in time (cf., e.g., [17]) in which the temperature $u(x, t), 0 \leq t<T$, has to be determined (for any fixed $t \in[0, T)$ ), while (noisy) temperature data for $u(x, T)$ are given and $u=u(x, t)$ satisfies the evolution equation

$$
u_{t}+L u=0 \quad(0 \leq t<T)
$$

with operators $L$ as in Section 1. If we formulate this backward heat equation problem as an operator equation $A u(x, t)=u(x, T), A: H=L^{2}(\Omega) \rightarrow L^{2}(\Omega)$, and if the smoothness condition

$$
u(x, t) \in M=\left\{u(x, t) \in H \mid\|u(x, 0)\|_{p} \leq E, p \geq 0\right\}
$$

is assumed, then this condition can be reformulated into an equivalent condition $u(x, t) \in$ $M_{\varphi, E}$ with $\varphi$ given by

$$
\varphi(\lambda)=\lambda^{\frac{1}{T-t}}\left[\frac{1}{2(T-t)} \ln \frac{1}{\lambda}\right]^{-p} .
$$

Now Theorem 2.2 (cf. below) can be applied and one obtains that the temperature $u(x, t)$ depends in a Hölder continuous way on the final data $u(x, T)$ for any $t \in(0, T)$, and in a logarithmic way in the case $t=0, p>0$. For the special case $t \in(0, T)$ and $p=0$ this result can also be obtained by applying the 'method of logarithmic convexity' (cf., e.g., $[2]$ ).

For our formulated problems (P1) and (P2) in Section 1 we will see in Sections 3 and 4 that the specific a priori restriction (1.5) on the unknown solution can be reformulated into equivalent conditions (2.3) with special functions $\varphi=\varphi(\lambda)$ that can be given in parameter representation. This reformulation enables us to apply optimality results known for 'general' source sets (2.3).

In order to derive explicit (best possible) error bounds for the worst case error $\Delta(\delta, R)$ defined in (2.2) and in order to obtain optimality results for special regularization methods we assume in this section the following.

Assumption 2.1. The function $\varphi:(0, a) \rightarrow(0, \infty)$ in $(2.3)$ (where $a$ is a constant with $\left.\left\|A^{*} A\right\| \leq a\right)$ is continuous and satisfies

(i) $\lim _{\lambda \rightarrow 0} \varphi(\lambda)=0$

(ii) $\varphi(\lambda)$ is strong monotonically increasing on $(0, a)$

(iii) $\rho(\lambda)=\lambda \varphi^{-1}(\lambda):(0, \varphi(a)] \rightarrow(0, a \varphi(a)]$ is convex.

The following theorem gives a formula for the best possible worst case error inf $R \Delta(\delta, R)$. The proof of this formula can be found in [17] and follows some ideas given in [19] (where the case $\varphi(\lambda)=\lambda^{p} \quad(p>0)$ is treated) and some ideas given in [13: Theorem 2.10]. 
Theorem 2.2. Let $M_{\varphi, E}$ be given by (2.3), let Assumption 2.1 be satisfied and let $\frac{\delta^{2}}{E^{2}} \in \sigma\left(A^{*} A \varphi\left(A^{*} A\right)\right)$ where $\sigma\left(A^{*} A\right)$ denotes the spectrum of the operator $A^{*} A$. Then

$$
\inf _{R} \Delta(\delta, R)=E \sqrt{\rho^{-1}\left(\frac{\delta^{2}}{E^{2}}\right)} .
$$

Note that the condition $\frac{\delta^{2}}{E^{2}} \in \sigma\left(A^{*} A \varphi\left(A^{*} A\right)\right)$ can only hold (for sufficiently small $\delta)$ provided problem (2.1) is ill-posed, which means in the compact case that the eigenvalues $\lambda_{i}$ of the operator $A^{*} A$ tend to zero. For well-posed problems (2.1) condition $\frac{\delta^{2}}{E^{2}} \in \sigma\left(A^{*} A \varphi\left(A^{*} A\right)\right)$ can never hold for sufficiently small $\delta$, hence this condition excludes the class of well-posed problems.

Furthermore, in [17] there has been proved that there are special regularization methods that realize the best possible worst case error given on the right-hand side of (2.4). In the following considerations we review two special methods: the method of generalized Tikhonov regularization and the method of generalized singular value decomposition.

In the method of generalized Tikhonov regularization a regularized approximation $x_{\alpha}^{\delta}$ is determined by solving the minimization problem

$$
\min _{x \in X} J_{\alpha}(x), \quad J_{\alpha}(x)=\left\|A x-y^{\delta}\right\|^{2}+\alpha\left\|\left[\varphi\left(A^{*} A\right)\right]^{-\frac{1}{2}} x\right\|^{2}
$$

or, equivalently, by solving the Euler equation

$$
\left(A^{*} A+\alpha\left[\varphi\left(A^{*} A\right)\right]^{-1}\right) x_{\alpha}^{\delta}=A^{*} y^{\delta} .
$$

This method appears to be optimal on the set $M_{\varphi, E}$ given by (2.3) provided the regularization parameter $\alpha$ is chosen properly. For this method the following statement holds (cf. [17]).

Theorem 2.3. Let $M_{\varphi, E}$ be given by (2.3), let Assumption 2.1 be satisfied, $\varphi(\lambda)$ : $(0, a] \rightarrow \mathbb{R}$ be two times differentiable, $\rho(\lambda)$ be strong convex on $(0, \varphi(a)]$ and $\frac{\delta^{2}}{E^{2}} \leq$ $a \varphi(a)$. If the regularization parameter $\alpha$ is chosen optimally by

$$
\alpha=\frac{\lambda}{\varphi^{-1}(\lambda) \varphi^{\prime}\left(\varphi^{-1}(\lambda)\right)}\left(\frac{\delta}{E}\right)^{2} \quad \text { with } \lambda=\rho^{-1}\left(\frac{\delta^{2}}{E^{2}}\right),
$$

then for the Tikhonov regularized solution $x_{\alpha}^{\delta}=R_{\alpha} y^{\delta}$ defined by (2.5) the optimal error estimate

$$
\Delta\left(\delta, R_{\alpha}\right) \leq E \sqrt{\rho^{-1}\left(\frac{\delta^{2}}{E^{2}}\right)}
$$

holds.

Proof ideas. From [19: Lemma 2.2] we have

$$
\begin{aligned}
\Delta\left(\delta, R_{\alpha}\right) & =\inf _{0<\xi<1}\left\|\frac{E^{2}}{\xi}\left(I-R_{\alpha} A\right) \varphi\left(A^{*} A\right)\left(I-R_{\alpha} A\right)^{*}+\frac{\delta^{2}}{1-\xi} R_{\alpha} R_{\alpha}^{*}\right\|^{\frac{1}{2}} \\
& =\inf _{0<\xi<1} \sup _{\lambda>0}\left\{\frac{E^{2} \alpha^{2} \varphi(\lambda)}{\xi[\lambda \varphi(\lambda)+\alpha]^{2}}+\frac{\delta^{2} \lambda \varphi^{2}(\lambda)}{(1-\xi)[\lambda \varphi(\lambda)+\alpha]^{2}}\right\}^{\frac{1}{2}} .
\end{aligned}
$$


Now we search for a stationary point $\left(\alpha_{0}, \xi_{0}, \lambda_{0}\right)$ of the expression $\{\ldots\}$ as a function of $\alpha, \xi$ and $\lambda$ and find $\alpha_{0}$ as given in (2.7) and $\xi_{0}=\frac{E^{2} \alpha_{0}}{\delta^{2}+E^{2} \alpha_{0}}$. We substitute $\alpha_{0}$ and $\xi_{0}$ into the expression $\{\ldots\}$ and prove that this expression as a function of $\lambda>0$ is bounded by $E \sqrt{\rho^{-1}\left(\frac{\delta^{2}}{E^{2}}\right)}$

Now let us review the method of generalized singular value decomposition. We describe this method for the important case of compact operators $A$ (although this method can be adapted also to the non-compact case). In this method a regularized approximation $x_{\alpha}^{\delta}$ is determined by

$$
\begin{aligned}
x_{\alpha}^{\delta} & =\sum_{s_{i} \geq \sqrt{\alpha}} \frac{\left(y^{\delta}, v_{i}\right)}{s_{i}} u_{i}+\frac{1}{\alpha} \sum_{s_{i}<\sqrt{\alpha}} s_{i}\left(y^{\delta}, v_{i}\right) u_{i} \\
& =\sum_{s_{i} \geq \sqrt{\alpha}} \frac{\left(y^{\delta}, v_{i}\right)}{s_{i}} u_{i}+\frac{1}{\alpha}\left\{A^{*} y^{\delta}-\sum_{s_{i} \geq \sqrt{\alpha}} s_{i}\left(y^{\delta}, v_{i}\right) u_{i}\right\}
\end{aligned}
$$

where $\left\{s_{i}, u_{i}, v_{i}\right\}$ denotes the singular system of the (compact) operator $A \in \mathcal{L}(X, Y)$ satisfying $A u_{i}=s_{i} v_{i}$ and $A^{*} v_{i}=s_{i} u_{i}$. This method appears to be optimal on the set $M_{\varphi, E}$ given by (2.3) provided the regularization parameter $\alpha$ is chosen properly. For this method the following optimality result can be established (cf. [17]), where the ideas of proof are similar to those of Theorem 2.3.

Theorem 2.4. Let $M_{\varphi, E}$ be given by (2.3), let Assumption 2.1 be satisfied, $\varphi(\lambda)$ : $(0, a] \rightarrow \mathbb{R}$ be two times differentiable, $\rho(\lambda)$ be strong convex on $(0, \varphi(a)]$ and $\frac{\delta^{2}}{E^{2}} \leq$ $a \varphi(a)$. If the regularization parameter $\alpha$ is chosen optimally by

$$
\alpha=\frac{\varphi\left(\lambda_{0}\right)+\lambda_{0} \varphi^{\prime}\left(\lambda_{0}\right)}{\varphi^{\prime}\left(\lambda_{0}\right)} \quad \text { with } \lambda_{0} \varphi\left(\lambda_{0}\right)=\left(\frac{\delta}{E}\right)^{2} \text {, }
$$

then for the regularized solution $x_{\alpha}^{\delta}=R_{\alpha} y^{\delta}$ defined by (2.9) the optimal error estimate (2.8) holds.

\section{Optimal error bounds for problem (P1)}

In this section we consider the elliptic problem

$$
\left.\begin{array}{cl}
u_{t t}-L u=0 & \text { for } 0<t \leq T \text { and } x \in \Omega \subset \mathbb{R}^{n} \\
u_{t}(x, 0)=0 & \text { for } x \in \Omega
\end{array}\right\}
$$

and treat the question concerning the best possibe worst case error for identifying $u(x, t)$ (for some fixed $t \in(0, T]$ ) from noisy data $u^{\delta}(x, 0)$ provided $\left\|u(x, 0)-u^{\delta}(x, 0)\right\| \leq \delta$ and $u(x, t) \in M_{p, E}$ where $M_{p, E}$ is given by (1.5). Let us formulate the problem of identifying $u(x, t)$ from (unperturbed) data $u(x, 0)$ as operator equation

$$
A(t) u(x, t)=u(x, 0)
$$


with linear operator $A(t) \in \mathcal{L}(H, H)$. By the Fourier method one has the unique solution

$$
u(x, t)=\sum_{i=1}^{\infty}\left(u(x, 0), u_{i}\right) \cosh \left(\sqrt{l_{i}} t\right) u_{i}
$$

of problem (3.1), consequently,

$$
\left(u(x, 0), u_{i}\right)=\frac{\left(u(x, t), u_{i}\right)}{\cosh \left(\sqrt{l_{i}} t\right)}
$$

which shows us that the operator $A(t): H \rightarrow H$ of problem (3.2) has the representation

$$
A(t) u(x, t)=\sum_{i=1}^{\infty} \frac{\left(\dot{u}(x, t), u_{i}\right)}{\cosh \left(\sqrt{l_{i}} t\right)} u_{i} .
$$

Hence $A(t) \in \mathcal{L}(H, H)$ can be written in the form

$$
A(t)=\frac{1}{\cosh (\sqrt{L} t)}
$$

We realize that $A(t): H \rightarrow H$ is a linear self-adjoint compact operator with eigenvalues $s_{i}=\frac{1}{\cosh \left(\sqrt{T_{i}}\right)}$ and eigenelements $u_{i}$. Since $s_{i}$ decay exponentially fast we realize that problem (3.2) is severely ill-posed. The ill-posedness becomes worse as $t$ increases.

Now let us reformulate condition (1.5) into an equivalent one of form (2.3) with a special function $\varphi=\varphi(\lambda)$.

Proposition 3.1. Consider the operator equation (3.2). Then the set $M_{p, E}$ given in (1.5) is equivalent to the general source set $M_{\varphi, E}$ given in (2.3) provided $\varphi=\varphi(\lambda)$ is given (in parameter representation) by

$$
\left.\begin{array}{l}
\lambda(l)=\frac{1}{\cosh ^{2}(\sqrt{l} t)} \\
\varphi(l)=l^{-p} \frac{\cosh ^{2}(\sqrt{l} t)}{\cosh ^{2}(\sqrt{l} T)}
\end{array}\right\} \quad\left(l_{1} \leq l<\infty\right) .
$$

Proof. From $A(t) u(x, t)=u(x, 0)$ for $0<t \leq T$ we have

which gives

$$
\left(u(x, 0), u_{i}\right)=\frac{\left(u(x, t), u_{i}\right)}{\cosh \left(\sqrt{l_{i}} t\right)}=\frac{\left(u(x, T), u_{i}\right)}{\cosh \left(\sqrt{l_{i}} T\right)}
$$

$$
u(x, T)=\frac{\cosh (\sqrt{L} T)}{\cosh (\sqrt{L} t)} u(x, t)
$$

Hence, the inequality $\|u(x, T)\|_{p} \leq E$ is equivalent to the inequality

$$
\left\|L^{\frac{p}{2}} \frac{\cosh (\sqrt{L} T)}{\cosh (\sqrt{L} t)} u(x, t)\right\| \leq E
$$

which shows us that the operator function $\varphi\left(A^{*} A\right)$ in (2.3) has the representation

$$
\varphi\left(A^{*} A\right)=L^{-p} \frac{\cosh ^{2}(\sqrt{L} t)}{\cosh ^{2}(\sqrt{L} T)} .
$$

Together with (3.3) we obtain that $\varphi$ is given by (3.4) 
The function $\varphi=\varphi(\lambda)\left(\lambda \in\left(0, \frac{1}{\cosh ^{2}(\sqrt{1} t)}\right]\right)$ which is defined in parameter representation by (3.4) possesses the following properties.

Proposition 3.2. The function $\varphi=\varphi(\lambda)$ defined by (3.4) is continuous and satisfies the properties:

(i) $\lim _{\lambda \rightarrow 0} \varphi(\lambda)=0$.

(ii) $\varphi(\lambda)$ is strong monotonically increasing.

(iii) $\rho(\lambda)=\lambda \varphi^{-1}(\lambda)$ is strong monotonically increasing and possesses the parameter. representation

$$
\left.\begin{array}{l}
\lambda(l)=l^{-p} \frac{\cosh ^{2}(\sqrt{l} t)}{\cosh ^{2}(\sqrt{l} T)} \\
\rho(l)=l^{-p} \frac{1}{\cosh ^{2}(\sqrt{l} T)}
\end{array}\right\} \quad\left(l_{1} \leq l<\infty\right)
$$

(iv) $\rho^{-1}(\lambda)$ is strong monotonically increasing and possesses the parameter representation

$$
\left.\begin{array}{rl}
\lambda(l) & =l^{-p} \frac{1}{\cosh ^{2}(\sqrt{l} T)} \\
\rho^{-1}(l) & =l^{-p} \frac{\cosh ^{2}(\sqrt{l} t)}{\cosh ^{2}(\sqrt{l} T)}
\end{array}\right\} \quad\left(l_{1} \leq l<\infty\right) .
$$

(v) For the inverse function $\rho^{-1}$ of $\rho$ there holds for any fixed $t \in(0, T]$

$$
\rho^{-1}(\lambda)=\left(\frac{\lambda}{4}\right)^{1-\frac{1}{T}}\left[\frac{1}{T} \ln \frac{1}{\sqrt{\lambda}}\right]^{-2 p \frac{l}{T}}(1+o(1)) \quad \text { for } \lambda \rightarrow 0 .
$$

Proof. Consider $\lambda(l)$ given by (3.4). From

$$
\dot{\lambda}=-\frac{t \sinh (\sqrt{l} t)}{\sqrt{l} \cosh ^{3}(\sqrt{l} t)}<0
$$

we realize that $\lambda(l)$ is strong monotonically decreasing with $\lim _{l \rightarrow \infty} \lambda(l)=0$. Consequently,

$$
\lim _{\lambda \rightarrow 0} \varphi(\lambda)=\lim _{l \rightarrow \infty} l^{-p} \frac{\cosh ^{2}(\sqrt{l} t)}{\cosh ^{2}(\sqrt{l} T)}=0,
$$

hence assertion (i) is proved. From

$$
\dot{\varphi}(l)=-\frac{\cosh ^{2}(\sqrt{l} t)}{l^{p+1} \cosh ^{2}(\sqrt{l} T)}\{p-\sqrt{l} t \tanh (\sqrt{l} t)+\sqrt{l} T \tanh (\sqrt{l} T)\}<0
$$

(note that $f(t)=t \tanh t$ is strong monotonically increasing) we obtain together with $\varphi^{\prime}(\lambda)=\frac{\varphi(l)}{\dot{\lambda}(l)}$ and $\dot{\lambda}(l)<0$ that assertion (ii) holds. From assertions (i) and (ii) it follows that $\varphi^{-1}(\lambda)$ is strong monotonically increasing, consequently, $\rho(\lambda)$ is strong 
monotonically increasing, where (3.6) follows from the parameter representation of $\varphi^{-1}$ which is given by

$$
\left.\begin{array}{rl}
\lambda(l) & =l^{-p} \frac{\cosh ^{2}(\sqrt{l} t)}{\cosh ^{2}(\sqrt{l} T)} \\
\varphi^{-1}(l) & =\frac{1}{\cosh ^{2}(\sqrt{l} t)}
\end{array}\right\} \quad\left(l_{1} \leq l<\infty\right) .
$$

Now assertion (iv) is a direct consequence of assertion (iii). In order to prove assertion (v) we show that $\lim _{\lambda \rightarrow 0} F(\lambda)=1$ where $F(\lambda)$ is given by

$$
F(\lambda)=\rho^{-1}(\lambda)\left(\frac{4}{\lambda}\right)^{1-\frac{1}{T}}\left[\frac{1}{T} \ln \frac{1}{\sqrt{\lambda}}\right]^{2 p \frac{t}{T}}
$$

We use (3.7), note that $\lambda(l)$ is strong monotonically decreasing with $\lim _{l \rightarrow \infty} \lambda(l)=0$ and obtain together with

$$
\lim _{l \rightarrow \infty}\left[4 \cosh ^{2}(\sqrt{l} T)\right]^{1-\frac{\iota}{T}} \frac{\cosh ^{2}(\sqrt{l} t)}{\cosh ^{2}(\sqrt{l} T)}=1
$$

that

$$
\begin{aligned}
\lim _{\lambda \rightarrow 0} F(\lambda) & =\lim _{l \rightarrow \infty} l^{-p} \frac{\cosh ^{2}(\sqrt{l} t)}{\cosh ^{2}(\sqrt{l} T)}\left[4 l^{p} \cosh ^{2}(\sqrt{l} T)\right]^{1-\frac{t}{T}}\left[\frac{1}{T} \ln \left\{l^{\frac{p}{2}} \cosh (\sqrt{l} T)\right\}\right]^{2 p \frac{l}{T}} \\
& =\lim _{l \rightarrow \infty}\left[\frac{1}{\sqrt{l} T} \ln \left\{l^{\frac{p}{2}} \cosh (\sqrt{l} T)\right\}\right]^{2 p \frac{t}{T}} \\
& =1
\end{aligned}
$$

and the assertion is proved

Remark 3.3. Note that in case $p=0$ the function $\rho^{-1}$ can be given explicitly. In this case we obtain from (3.7) that

$$
\rho^{-1}(\lambda)=\lambda\left[\cosh \left(\frac{t}{T} \operatorname{arcosh} \frac{1}{\sqrt{\lambda}}\right)\right]^{2} .
$$
convex.

In our next proposition we formulate conditions under which the function $\rho$ is strong

Proposition 3.4. The function $\rho$ defined by (3.6) is strong convex if and only if

$$
p^{2}+\psi_{1}\left(\sqrt{l_{1}} T\right) p+\psi_{2}\left(\sqrt{l_{1}} T\right)>0
$$

holds where the functions $\psi_{1}$ and $\psi_{2}$ with $\tau=\frac{t}{T}$ are given by

$$
\begin{aligned}
& \psi_{1}(x)=2 x \tanh x-\tau x \operatorname{coth}(2 \tau x)-\frac{1}{2} \\
& \psi_{2}(x)=x \tanh x[x \operatorname{coth}(2 x)-\tau x \operatorname{coth}(2 \tau x)]
\end{aligned}
$$


Proof. From $\rho^{\prime \prime}=\frac{\vec{\rho} \dot{\lambda}-\dot{\phi} \bar{\lambda}}{\dot{\lambda}^{3}}$ and $\dot{\lambda}<0$ we obtain that $\rho^{\prime \prime}>0$ is equivalent to $\ddot{\rho} \dot{\lambda}<\dot{\rho} \ddot{\lambda}$. Note that $\lambda(l)=\rho(l) r(l)$ with $r(l)=\cosh ^{2}(\sqrt{l} t)$, hence the inequality $\rho^{\prime \prime}>0$ is equivalent to the inequality

$$
\rho \ddot{\rho}-2 \dot{\rho}^{2}<\rho \dot{\rho} \frac{\ddot{r}}{\dot{r}}
$$

We substitute $x=\sqrt{l} T$, introduce the constant $\tau=\frac{t}{T}$ and obtain by .elementary calculations that (3.15) is equivalent to the inequality

$$
p^{2}+\psi_{1}(x) p+\psi_{2}(x)>0
$$

Consequently, $\rho$ is strong convex if and only if (3.16) holds for all $x \in\left[\sqrt{l_{1}} T, \infty\right)$. Now it can be shown that both functions $\psi_{1}$ and $\psi_{2}$ are monotonically increasing on $\mathbb{R}^{+}$for any fixed $\tau \in(0,1]$. Consequently, $\rho$ is strong convex if and only if (3.12) holds

Remark 3.5. Since the function $g(x)=x \operatorname{coth}(2 x)$ is strong monotonically increasing on $\mathbb{R}^{+}$we find that $\psi_{2}(x)>0$ for $x>0$ and $0 \leq \tau<1$, consequently, in the special case $p=0$ we have the following result:

(i) Let $p=0$ and $t<T$. Then the function $\rho$ defined by (3.6) is strong convex.

Furthermore, in the special case $t=T$ (i.e. $\tau=1$ ) we have $\psi_{2}(x)=0$, consequently, from Proposition 3.4 we conclude in this case the following result:

(ii) Let $p>0$ and $t=T$ (i.e. $\tau=1$ ). Then the function $\rho$ defined by (3.6) is strong convex provided

$$
p>\frac{1}{2}+\sqrt{l_{1}} T \operatorname{coth}\left(2 \sqrt{l_{1}} T\right)-2 \sqrt{l_{1}} T \tanh \left(\sqrt{l_{1}} T\right)
$$

Since the function $\psi_{1}$ with $\tau=1$ is strong monotonically increasing with $\lim _{x \rightarrow+0} \psi_{1}(x)$ $=-1$ and $\psi_{1}\left(x_{0}\right)=0$ for $x_{0} \in(1.00955,1.00956)$ we obtain:

(iii) Inequality (3.17) is satisfied for all $\sqrt{l_{1}} T>0$ provided $p \geq 1$ holds.

(iv) Inequality (3.17) is satisfied for all $p>0$ provided $\sqrt{l_{1}} T \geq 1.01$ holds.

Finally, let us discuss the case $p>0$ and $t<T$ (i.e. $0 \leq \tau<1$ ). In this case we conclude from the valid inequality $x \operatorname{coth}(2 x) \geq \tau x \operatorname{coth}(2 \tau x)$ for $x \geq 0$ that (3.16) is satisfied provided $p^{2}+p[2 x \tanh x-x \operatorname{coth}(2 x)-0.5]>0$ holds, consequently there holds the following result:

(v) Let $p>0$ and $t<T$. Then the function $\rho$ defined by (3.6) is strong convex provided (3.17) holds.

Now we are in a position to formulate our main result of this section concerning the best possible worst case error $\omega(\delta, t)$ defined in (1.7) for identifying the solution $u(x, t)$ of problem (3.1) from noisy data $u^{\delta}(x, 0)$ under the conditions $\left\|u(x, 0)-u^{\delta}(x, 0)\right\| \leq \delta$ and $u(x, t) \in M_{p, E}$ where the set $M_{p, E}$ is given by (1.5). We apply Theorems 2.2 and 2.3 , use Propositions 3.2 and 3.4 as well as Remarks 3.3 and 3.5 , and obtain 
Theorem 3.6. Let $\frac{\delta^{2}}{E^{2}}$ be an eigenvalue of the operator $\frac{L^{-p}}{\cosh ^{2}(\sqrt{L} T)}$. Then:

(i) In case $p=0$ and $0<t<T$ there holds

$$
\omega(\delta, t)=\delta \cosh \left(\frac{t}{T} \operatorname{arcosh} \frac{E}{\delta}\right)=E^{\frac{t}{T}}\left(\frac{\delta}{2}\right)^{1-\frac{t}{T}}(1+o(1)) \text { for } \delta \rightarrow 0
$$

(Hölder stability).

(ii) In case $p>0$ and $t=T$ there holds under condition (3.17)

$$
\omega(\delta, T)=E l_{0}^{-\frac{p}{2}}=E\left[\frac{1}{T} \ln \frac{1}{\delta}\right]^{-p}(1+o(1)) \text { for } \delta \rightarrow 0
$$

(logarithmic stability), where $l_{0}=l_{0}(\delta)$ denotes the unique solution of the equation $l^{\frac{2}{2}} \cosh (\sqrt{l} T)=\frac{E}{\delta}$.

(iii) In case $p>0$ and $0<t<T$ there holds under condition (3.12)

$$
\begin{aligned}
\omega(\delta, t) & =E l_{0}^{-\frac{R}{2}} \frac{\cosh \left(\sqrt{l_{0}} t\right)}{\cosh \left(\sqrt{l_{0}} T\right)} \\
& =E^{\frac{l}{T}}\left(\frac{\delta}{2}\right)^{1-\frac{\varepsilon}{T}}\left[\frac{1}{T} \ln \frac{1}{\delta}\right]^{-p \frac{t}{T}}(1+o(1)) \text { for } \delta \rightarrow 0 .
\end{aligned}
$$

\section{Optimal error bounds for problem (P2)}

In this section we consider the elliptic problem

$$
\left.\begin{array}{cl}
u_{t t}-L u=0 & \text { for } 0<t \leq T \text { and } x \in \Omega \subset \mathbb{R}^{n} \\
u(x, 0)=0 & \text { for } x \in \Omega
\end{array}\right\}
$$

and treat the question concerning the best possibe worst case error for identifying $u(x, t)$ (for some fixed $t \in(0, T]$ ) from noisy data $u_{t}^{\delta}(x, 0)$ provided $\left\|u_{t}(x, 0)-u_{t}^{\delta}(x, 0)\right\| \leq \delta$ and $u(x, t) \in M_{p, E}$ where $M_{p, E}$ is given by (1.5). Let us formulate the problem of identifying $u(x, t)$ from (unperturbed) data $u_{t}(x, 0)$ as operator equation

$$
A(t) u(x, t)=u_{t}(x, 0) .
$$

Applying Fourier's method to problem (4.1) it can be shown (in analogy to Section 3) that $A(t) \in \mathcal{L}(H, H)$ has the representation

$$
A(t)=\frac{\sqrt{L}}{\sinh (\sqrt{L} t)} .
$$

We realize that $A(t): H \rightarrow H$ is a linear self-adjoint compact operator with eigenvalues $s_{i}=\frac{\sqrt{T_{i}}}{\sinh \left(\sqrt{T_{i}} t\right)}$ and eigenelements $u_{i}$. Since $s_{i}$ decay exponentially fast we realize that problem (4.2) is severely ill-posed. The ill-posedness becomes worse as $t$ increases.

In analogy to the proof of Proposition 3.1 we can reformulate condition (1.5) into an equivalent one of form (2.3) with a special function $\varphi=\varphi(\lambda)$. 
Proposition 4.1. Consider the operator equation (4.2). Then the set $M_{p, E}$ given in (1.5) is equivalent to the general source set $M_{\varphi, E}$ given in (2.3) provided $\varphi=\varphi(\lambda)$ is given (in parameter representation) by

$$
\left.\begin{array}{l}
\lambda(l)=\frac{l}{\sinh ^{2}(\sqrt{l} t)} \\
\varphi(l)=l^{-p} \frac{\sinh ^{2}(\sqrt{l} t)}{\sinh ^{2}(\sqrt{l} T)}
\end{array}\right\} \quad\left(l_{1} \leq l<\infty\right) .
$$

In analogy to the proof of Proposition 3.2 it can be shown that the function $\varphi=$ $\varphi(\lambda)\left(\lambda \in\left(0, \frac{l_{1}}{\sinh ^{2}(\sqrt{1} t)}\right]\right)$ defined by (4.4) possesses the following properties.

Proposition 4.2. The function $\varphi=\varphi(\lambda)$ defined by (4.4) is continuous and satisfies the properties:

(i) $\lim _{\lambda \rightarrow 0} \varphi(\lambda)=0$.

(ii) $\varphi(\lambda)$ is strong monotonically increasing.

(iii) $\rho(\lambda)=\lambda \varphi^{-1}(\lambda)$ is strong monotonically increasing and possesses the parameter representation

$$
\left.\begin{array}{l}
\lambda(l)=l^{-p} \frac{\sinh ^{2}(\sqrt{l} t)}{\sinh ^{2}(\sqrt{l} T)} \\
\rho(l)=l^{1-p} \frac{1}{\sinh ^{2}(\sqrt{l} T)}
\end{array}\right\} \quad\left(l_{1} \leq l<\infty\right) .
$$

(iv) $\rho^{-1}(\lambda)$ is strong monotonically increasing and possesses the parameter representation

$$
\left.\begin{array}{rl}
\lambda(l) & =l^{1-p} \frac{1}{\sinh ^{2}(\sqrt{l} T)} \\
\rho^{-1}(l) & =l^{-p} \frac{\sinh ^{2}(\sqrt{l} t)}{\sinh ^{2}(\sqrt{l} T)}
\end{array}\right\} \quad\left(l_{1} \leq l<\infty\right) .
$$

(v) For the inverse function $\rho^{-1}$ of $\rho$ there holds

$$
\rho^{-1}(\lambda)=\left(\frac{\lambda}{4}\right)^{1-\frac{t}{T}}\left[\frac{1}{T} \ln \frac{1}{\sqrt{\lambda}}\right]^{2(1-p) \frac{1}{T}-2}(1+o(1)) \quad \text { for } \lambda \rightarrow 0 .
$$

Remark 4.3. Note that in case $p=1$ the function $p^{-1}$ can be given explicitly. In this case we obtain from (4.6) that for any $t \in(0, T]$ there holds

$$
\rho^{-1}(\lambda)=\frac{\lambda\left[\sinh \left(\frac{t}{T} \operatorname{arsinh} \frac{1}{\sqrt{\lambda}}\right)\right]^{2}}{\left[\frac{1}{T} \operatorname{arsinh}\left(\frac{1}{\sqrt{\lambda}}\right)\right]^{2}} .
$$

In our next proposition we formulate conditions under which the function $\rho$ is strong convex. The proof of this result can be done in analogy to that of Proposition 3.4. 

only if

Proposition 4.4 The function $\rho=\rho(\lambda)$ defined by (4.5) is strong convex if and

$$
p^{2}+\psi_{1}\left(\sqrt{l_{1}} T\right) p+\psi_{2}\left(\sqrt{l_{1}} T\right)>0
$$

holds. In (4.9) the functions $\psi_{1}$ and $\psi_{2}$ are given by

$$
\begin{aligned}
& \psi_{1}(x)=2 x \operatorname{coth} x-1-g(\tau x) \\
& \psi_{2}(x)=(x \operatorname{coth} x-1)[g(x)-g(\tau x)]
\end{aligned}
$$

with $\tau=\frac{t}{T}$ and

$$
g(x)=\frac{x}{2} \operatorname{coth} x+\frac{x^{2}}{2(x \operatorname{coth} x-1)} .
$$

Remark 4.5. Since $\psi_{2}(x)>0$ for $x>0$ and $0 \leq \tau<1$ we obtain for the special case $p=0$ the following result: convex.

(i) Let $p=0$ and $t<T$. Then the function $\rho=\rho(\lambda)$ defined by (4.5) is strong

Furthermore, in the special case $t=T$ (i.e. $\tau=1$ ) we have $\psi_{2}(x)=0$, consequently, from Proposition 4.4 we conclude in this case the following result:

(ii) Let $p>0$ and $t=T$ (i.e. $\tau=1$ ). Then the function $\rho=\rho(\lambda)$ defined by (4.5) is strong. convex provided

$$
p>-\psi_{1}\left(\sqrt{l_{1}} T\right)
$$

Since the function $\psi_{1}$ with $\tau=1$ is strong monotonically increasing with $\lim _{x \rightarrow+0} \psi_{1}(x)$ $=-1$ and $\psi_{1}\left(x_{0}\right)=0$ for $x_{0} \in(1.74389,1.74390)$ we obtain the following:

(iii) Inequality (4.13) with $\tau=1$ is satisfied for all $\sqrt{l_{1}} T>0$ provided $p \geq 1$ holds.

(iv) Inequality (4.13) with $\tau=1$ is satisfied for all $p>0$ provided $\sqrt{l_{1}} T \geq 1.744$.

Finally, let us discuss the case $p>0$ and $t<T$ (i.e. $0 \leq \tau<1$ ). In this case we conclude from the valid inequality $g(x) \geq g(\tau x)$ for $x \geq 0$ that (4.9) is satisfied provided (4.13) holds with $\tau=1$, consequently there holds the following result:

(v) Let $p>0$ and $t<T$. Then the function $\rho=\rho(\lambda)$ defined by (4.5) is strong convex provided (4.13) holds with $\tau=1$.

Now we are in a position to formulate our main result of this section concerning the best possible worst case error $\omega(\delta, t)$ defined in (1.7) for identifying the solution $u(x, t)$ of problem (4.1) from noisy data $u_{t}^{\delta}(x, 0)$ under the conditions $\left\|u_{t}(x, 0)-u_{t}^{\delta}(x, 0)\right\| \leq \delta$ and $u(x, t) \in M_{p, E}$ where the set. $M_{p, E}$ is given by (1.5). We apply Theorems 2.2 and 2.3, use Propositions 4.2 and 4.4 as well as Remark 4.5 and obtain

Theorem 4.6. Let $\frac{\delta^{2}}{E^{2}}$ be an eigenvalue of the operator $\frac{L^{1-p}}{\sinh ^{2}(\sqrt{L} T)}$. Then:

(i) In case $p=0$ and $0<t<T$ there holds

$$
\omega(\delta, t)=E l_{0}^{-\frac{R}{2}} \frac{\sinh \left(\sqrt{l_{0}} t\right)}{\sinh \left(\sqrt{l_{0}} T\right)}=E^{\frac{\ell}{T}}\left[\frac{T \delta}{2 \ln \frac{1}{\delta}}\right]^{1-\frac{t}{T}}(1+o(1)) \text { for } \delta \rightarrow 0
$$


where $l_{0}$ is the (unique) solution of the equation $\frac{1^{1-p}}{\sinh ^{2}(\sqrt{i})}=\frac{\delta^{2}}{E^{2}}$ (Hölder stability).

(ii) In case $p>0$ and $t=T$ there holds under condition (4.13)

$$
\omega(\delta, T)=E l_{0}^{-\frac{\rho}{2}}=E\left[\frac{1}{T} \ln \frac{1}{\delta}\right]^{-p}(1+o(1)) \quad \text { for } \delta \rightarrow 0
$$

(logarithmic stability).

(iii) In case $p>0$ and $0<t<T$ there holds under the condition (4.9)

$$
\begin{aligned}
\omega(\delta, t) & =E l_{0}^{-\frac{p}{2}} \frac{\sinh \left(\sqrt{l_{0}} t\right)}{\sinh \left(\sqrt{l_{0}} T\right)} \\
& =E^{\frac{6}{T}}\left(\frac{\delta}{2}\right)^{1-\frac{2}{T}}\left[\frac{1}{T} \ln \frac{1}{\delta}\right]^{(1-p) \frac{t}{T}-1}(1+o(1)) \text { for } \delta \rightarrow 0 .
\end{aligned}
$$

Note that in case $p=1$ the best possible error bound $\omega(\delta, t)$ can be given explicitly. In this case we obtain from (4.8) that

$$
\omega(\delta, t)=\frac{\delta \dot{T} \sinh \left(\frac{t}{T} \operatorname{arsinh}\left(\frac{E}{\delta}\right)\right)}{\operatorname{arsinh}\left(\frac{E}{\delta}\right)} .
$$

Furthermore note that for $t<T$ the best possible error bounds for problem (P2) given in Theorem 4.6 are 'smaller' than those for problem (P1) given in Theorem 3.6.

\section{Optimal regularization methods for problem (P1)}

In Section 3 we have proved that the best possible worst case error for identifying $u(x, t)$ in (3.2) from noisy data $u^{\delta}(x, 0)$ under the conditions $\left\|u(x, 0)-u^{\delta}(x, 0)\right\| \leq \delta$ and $u(x, t) \in M_{p, E}$ (with $M_{p, E}$ given by (1.5)) is given by (3.18) - (3.20) (in cases $p=0$ and $0<t<T, p>0$ and $t=T$, and $p>0$ and $0<t<T$, respectively). From these results we realize that under the above conditions $\left\|u(x, 0)-u^{\delta}(x, 0)\right\| \leq \delta$ and $u(x, t) \in M_{p, E}$ there do not exist any methods $R(t): H \rightarrow H$ which guarantee an error bound for $\left\|R(t) u^{\delta}(x, 0)-u(x, t)\right\|$ which is smaller than those given on the right-hand side of (3.18) - (3.20), respectively.

In this section we consider the methods of generalized Tikhonov regularization and generalized singular value decomposition, apply both methods to problem (P1) and show how to choose the regularization parameter such that both methods guarantee the optimal error bounds given by (3.18) - (3.20), respectively. These optimality results will be obtained by applying Theorems 2.3 and 2.4 , respectively.

For problem (P1) we obtain together with (3.3) and (3.5) that the method of generalized Tikhonov regularization (2.5) consists in the determination of a regularized approximation $u_{\alpha}^{\delta}=u_{\alpha}^{\delta}(x, t)$ by solving the minimization problem

$$
\min _{u \in H} J_{\alpha}(u), \quad J_{\alpha}(u)=\left\|\frac{1}{\cosh (\sqrt{L} t)} u-u^{\delta}(x, 0)\right\|^{2}+\alpha\left\|L^{\frac{p}{2}} \frac{\cosh (\sqrt{L} T)}{\cosh (\sqrt{L} t)} u\right\|^{2} .
$$


Hence $u_{\alpha}^{\delta}=u_{\alpha}^{\delta}(x, t)$ is the solution of the Euler equation

$$
\left(\frac{1}{\cosh ^{2}(\sqrt{L} t)}+\alpha L^{p} \frac{\cosh ^{2}(\sqrt{L} T)}{\cosh ^{2}(\sqrt{L} t)}\right) u_{\alpha}^{\delta}(x, t)=\frac{1}{\cosh (\sqrt{L} t)} u^{\delta}(x, 0)
$$

of the functional $J_{\alpha}$, or equivalently, $u_{\alpha}^{\delta}=u_{\alpha}^{\delta}(x, t)$ is the solution of the symmetric, positive definite operator equation

$$
(A(t)+\alpha B(t)) u_{\alpha}^{\delta}(x, t)=u^{\delta}(x, 0)
$$

with $A(t)$ given by $(3.3)$ and $B(t)$ given by

$$
B(t)=L^{p} \frac{\cosh ^{2}(\sqrt{L} T)}{\cosh (\sqrt{L} t)}
$$

Note that (5.3) is the generalized Lavrentjev method for solving equation (3.2). The 'ordinary' Lavrentjev method (cf. [11]) is characterized by (5.3) with $\alpha B(t)$ replaced by $\alpha I$; in our method (5.3) we have a generalization in such a way that 'optimal' error bounds can be guaranteed. A further equivalent representation of (5.2) is given by

$$
A(t) u_{\alpha}^{\delta}(x, t)=(I+\alpha C)^{-1} u^{\delta}(x, 0)
$$

with $C=L^{p} \cosh ^{2}(\sqrt{L} T)$ which shows us that the regularized solution $u_{\alpha}^{\delta}(x, t)$ can be computed by the following steps:

(i) Given noisy data $u^{\delta}(x, 0)$, compute 'smoothed' data $u_{\alpha}^{\delta}(x, 0)$ by solving the symmetric positive definite operator equation

$$
(I+\alpha C) u_{\alpha}^{\delta}(x, 0)=u^{\delta}(x, 0) .
$$

(ii) Given 'smoothed' data $u_{\alpha}^{\delta}(x, 0)$ from step (i), solve the unregularized problem

$$
A(t) u_{\alpha}^{\delta}(x, t)=u_{\alpha}^{\delta}(x, 0),
$$

i.e. solve problem (3.1) with Cauchy data $u(x, 0)=u_{\alpha}^{\delta}(x, 0)$.

A similar regularization idea has been used by Hao (cf. [7]), where the data smoothing step (i) has been done by mollification techniques and where order optimal error bounds have been obtained.

Note that for any fixed $t=t_{0} \in(0, T]$ the computation of $u_{\alpha}^{\delta}\left(x, t_{0}\right)$ by directly solving the (well-posed) regularized problems (5.2), (5.3) or (5.4), respectively, is quite expensive (especially in cases $n \geq 2$ ) since the generation of the (discretized) operators $A\left(t_{0}\right), B\left(t_{0}\right)$ and $C$ requires the multiple solution of the 'direct' problem (3.1) with different boundary conditions, and since the corresponding (discretized) operators are dense. From the computational amount of view it seems to be better to use gradient type methods for minimizing Tikhonov's functional $J_{\alpha}$, e.g. the classical gradient method

$$
u_{k+1}=u_{k}-\gamma_{k}\left\{L^{-p} \frac{\cosh \left(\sqrt{L} t_{0}\right)}{\cosh ^{2}(\sqrt{L} T)}\left[\frac{1}{\cosh \left(\sqrt{L} t_{0}\right)} u_{k}-u^{\delta}(x, 0)\right]+\alpha u_{k}\right\}
$$


$\left(k=0,1, \ldots, u_{0}=0\right)$ with suitable steplength parameters $\gamma_{k}$, or some conjugate gradient type methods which turned out to be quite effective also for (ill-posed) large scale problems (cf., e.g., [6]). For integer p-values one step of the above gradient iteration can effectively be realized by executing following well-posed computational steps:

(a) Compute the element $w_{1}(x)=\left[\frac{1}{\cosh \left(\sqrt{L} t_{0}\right)}\right] u_{k}$ which can be obtained by $w_{1}(x)=$ $v(x, 0)$ where $v(x, t)$ is the solution of the well-posed problem

$$
\left.\begin{array}{rlrl}
v_{t t}-L v=0 & & \text { for } t \in\left(0, t_{0}\right) \text { and } x \in \Omega \subset \mathbb{R}^{n} \\
v_{t}(x, 0)=0 & & \text { for } x \in \Omega \\
v\left(x, t_{0}\right)=u_{k} & & \text { for } x \in \Omega .
\end{array}\right\}
$$

(b) Compute the element $w_{2}(x)=\left[\frac{\cosh \left(\sqrt{L} t_{0}\right)}{\cosh (\sqrt{L} T)}\right]\left[w_{1}(x)-u^{\delta}(x, 0)\right]$ which can be obtained by $w_{2}(x)=v\left(x, t_{0}\right)$ where $v(x, t)$ is the solution of the well-posed problem

$$
\left.\begin{array}{rlrl}
v_{t}-L v & =0 & & \text { for } t \in(0, T) \text { and } x \in \Omega \subset \mathbb{R}^{n} \\
v_{\imath}(x, 0) & =0 & & \text { for } x \in \Omega \\
v(x, T) & =w_{1}(x)-u^{\delta}(x, 0) & & \text { for } x \in \Omega .
\end{array}\right\}
$$

(c) Compute the element $w_{3}(x)=\left[\frac{1}{\cosh (\sqrt{L} T)}\right] w_{2}(x)$ which can be obtained by $w_{3}(x)=v(x, 0)$ where $v(x, t)$ is the solution of problem $(5.6)$ with $w_{1}(x)-u^{\delta}(x, 0)$ replaced by $w_{2}(x)$.

(d) Compute $w_{4}(x)=L^{-p} w_{3}(x)$ by solving the problem $L^{p} w_{4}(x)=w_{3}(x)$.

(e) Compute $u_{k+1}=u_{k}-\gamma_{k}\left\{w_{4}(x)+\alpha u_{k}\right\}$.

In our following considerations we are going to apply Theorem 2.3 to problem (P1) and start our discussions with a proposition that shows us how to compute the optimal regularization parameter (2.7) provided the function $\varphi=\varphi(\lambda)$ is given in parameter representation.

Proposition 5.1. Let the function $\varphi=\varphi(\lambda)$ in Theorem 2.3 be given in parameter. representation

$$
\left.\begin{array}{l}
\lambda(l)=\psi_{1}(l) \\
\varphi(l)=\psi_{2}(l)
\end{array}\right\} \quad\left(l_{1} \leq l<\infty\right)
$$

Then for the optimal regularization parameter (2.7) there holds

$$
\alpha=\frac{\dot{\psi}_{1}\left(l_{0}\right) \psi_{2}\left(l_{0}\right)}{\psi_{1}\left(l_{0}\right) \dot{\psi}_{2}\left(l_{0}\right)}\left(\frac{\delta}{E}\right)^{2}
$$

where $l_{0}$ is the (unique) solution of the equation $\psi_{1}(l) \psi_{2}(l)=\frac{\delta^{2}}{E^{2}}$.

Proof. From (5.7) we have for the functions $\varphi^{-1}=\varphi^{-1}(\lambda)$ and $\rho=\rho(\lambda)$ the parameter representations

$$
\left.\begin{array}{rl}
\lambda(l) & =\psi_{2}(l) \\
\varphi^{-1}(l) & =\psi_{1}(l)
\end{array}\right\} \quad\left(l_{1} \leq l<\infty\right)
$$


and

$$
\left.\begin{array}{l}
\lambda(l)=\psi_{2}(l) \\
\rho(l)=\psi_{1}(l) \psi_{2}(l)
\end{array}\right\} \quad\left(l_{1} \leq l<\infty\right)
$$

Denote by $\lambda_{0}$ the (unique) solution of the equation $\rho(\lambda)=\frac{\delta^{2}}{E^{2}}$. Due to $(5.10)$, this solution can be found from the following two steps:

(a) Determine $l_{0}$ from the equation $\psi_{1}(l) \psi_{2}(l)=\frac{\delta^{2}}{E^{2}}$.

(b) Compute $\lambda_{0}=\psi_{2}\left(l_{0}\right)$.

Now from (b) and (5.9) we have $\varphi^{-1}\left(\lambda_{0}\right)=\psi_{1}\left(l_{0}\right)$ and together with (5.7) we conclude that

$$
\varphi^{\prime}\left(\varphi^{-1}\left(\lambda_{0}\right)\right)=\varphi^{\prime}\left(\psi_{1}\left(l_{0}\right)\right)=\frac{\dot{\psi}_{2}\left(l_{0}\right)}{\dot{\psi}_{1}\left(l_{0}\right)}
$$

Consequently, the desired formula (5.8) follows from (2.7) together with (a) and (b), the equation $\varphi^{-1}\left(\lambda_{0}\right)=\psi_{1}\left(l_{0}\right)$ and $(5.11)$

Theorem 5.2. Consider the operator equation (3.2) and suppose that its unknown solution $u(x, t)$ belongs to the set $M_{p, E}$ given by (1.5). If $(3.12)$ and $\frac{\delta^{2}}{E^{2}} \leq \frac{l_{1}^{-p}}{\cosh ^{2}\left(\sqrt{T_{1}} T\right)}$ hold, then the method of generalized Tikhonov regularization (5.1) is optimal on the set $M_{p, E}$ provided the regularization parameter $\alpha$ is chosen optimally by

$$
\alpha_{0}=\frac{\sqrt{l_{0}} t \tanh \left(\sqrt{l_{0}} t\right)}{p+\sqrt{l_{0}} T \tanh \left(\sqrt{l_{0}} T\right)-\sqrt{l_{0}} t \tanh \left(\sqrt{l_{0}} t\right)}\left(\frac{\delta}{E}\right)^{2}
$$

where $l_{0}$ is the (unique) solution of the equation

$$
l^{\frac{p}{2}} \cosh (\sqrt{l} T)=\frac{E}{\delta}
$$

In the case $t=T$ and $p>0$ there holds

$$
\alpha_{0}=\frac{1}{p}\left[\ln \frac{E}{\delta}\right]\left(\frac{\delta}{E}\right)^{2}(1+o(1)) \quad \text { for } \delta \rightarrow 0
$$

and in the case $0 \leq t<T$ and $p \geq 0$ there holds

$$
\alpha_{0}=\frac{t}{T-t}\left(\frac{\delta}{E}\right)^{2}(1+o(1)) \quad \text { for } \delta \rightarrow 0
$$

Furthermore, there holds the optimal error estimate $\left\|u_{\alpha_{0}}^{\delta}(x, t)-u(x, t)\right\| \leq \omega(\delta, t)$ where $\omega(\delta, t)$ is given by $(3.18)-(3.20)$, respectively.

Proof. The result of Theorem 5.2 follows from Theorems 2.3 and 3.6 where it remains to show that $(5.12)-(5.15)$ hold. The representations (5.12) and (5.13) follow 
from (2.7) and Proposition 5.1 by straight forward calculations and use of (3.9) and (3.10). In the case $t=T$ and $p>0$ we obtain

$$
\alpha_{0}=\frac{\sqrt{l_{0}} T}{p} \tanh \left(\sqrt{l_{0}} T\right)\left(\frac{\delta}{E}\right)^{2}
$$

where $l_{0}$ is the solution of (5.13), or equivalently, $\lambda_{0}$ is the solution of $\rho(\lambda)=\frac{\delta^{2}}{\bar{E}^{2}}$ where $\lambda_{0}$ and $l_{0}$ are related by $\lambda_{0}=l_{0}^{-p}$. Now we apply (3.8) and obtain

$$
\lambda_{0}=\rho^{-1}\left(\frac{\delta^{2}}{E^{2}}\right)=\left[\frac{1}{E} \ln \frac{E}{\delta}\right]^{-2 p}(1+o(1)) \quad \text { for } \delta \rightarrow 0 .
$$

Consequently, since $l_{0}=\lambda_{0}^{-\frac{1}{p}}$,

$$
\sqrt{l_{0}} T=\left[\ln \frac{E}{\delta}\right](1+o(1)) \quad \text { for } \delta \rightarrow 0 .
$$

Furthermore, since $l_{0} \rightarrow \infty$ for $\delta \rightarrow 0$ we have $\tanh \left(\sqrt{l_{0}} T\right) \rightarrow 1$ for $\delta \rightarrow 0$ which together with (5.16) and (5.17) yields (5.14). In the case $0 \leq t<T$ and $p \geq 0$ we proceed as follows: for $\delta \rightarrow 0$ there holds $l_{0} \rightarrow \infty$. Consequently, $\tanh \left(\sqrt{l_{0}} t\right) \rightarrow 1$ and $\tanh \left(\sqrt{l_{0}} T\right) \rightarrow 1$ for $\delta \rightarrow 0$ which together with (5.12) yields $(5.15)$

In the second part of this section we consider the method of generalized singular value decomposition, apply this method to problem (P1) and show by using Theorem 2.4 how to choose the regularization parameter such that this method is optimal on the set $M_{p, E}$.

For problem (P1) we obtain together with (3.3) and (3.5) that the method of generalized singular value decomposition (2.9) consists in the determination of a regularized approximation $u_{\alpha}^{\delta}(x, t)$ according to

$$
\begin{aligned}
u_{\alpha}^{\delta}(x, t) & =\sum_{s_{i} \geq \sqrt{\alpha}} \frac{\left(u^{\delta}(x, 0), u_{i}\right)}{s_{i}} u_{i}+\frac{1}{\alpha} \sum_{s_{i}<\sqrt{\alpha}} s_{i}\left(u^{\delta}(x, 0), u_{i}\right) u_{i} \\
& =\sum_{s_{i} \geq \sqrt{\alpha}} \frac{\left(u^{\delta}(x, 0), u_{i}\right)}{s_{i}} u_{i}+\frac{1}{\alpha}\left\{A(t) u^{\delta}(x, 0)-\sum_{s_{i} \geq \sqrt{\alpha}} s_{i}\left(u^{\delta}(x, 0), u_{i}\right) u_{i}\right\}
\end{aligned}
$$

with $s_{i}=\frac{1}{\cosh \left(\sqrt{t_{i}} t\right)}$ and $A(t)=\frac{1}{\cosh (\sqrt{L} t)}$. The element $w(x)=A\left(t_{0}\right) u^{\delta}(x, 0)$ can be obtained by $w(x)=v(x, 0)$ where $v(x, t)$ is the solution of problem (5.5) with $u_{k}$ replaced by $u^{\delta}(x, 0)$. Method (5.18) appears to be very cheap concerning the computational amount of work provided the eigenvalues and eigenelements of the operator $L$ are known analytically. Note that in the second representation of (5.18) only 'finite' sums occur.

In our following considerations we are going to apply Theorem 2.4 to problem (P1) and start our discussions with a proposition that shows us how to compute the optimal regularization parameter (2.10) provided the function $\varphi=\varphi(\lambda)$ possesses the parameter representation (5.7). 
Proposition 5.3. Let the function $\varphi=\varphi(\lambda)$ in Theorem 2.4 be given in parameter representation (5.7). Then for the optimal regularization parameter (2.10) there holds

$$
\alpha=\frac{\dot{\psi}_{1}\left(l_{0}\right) \psi_{2}\left(l_{0}\right)+\psi_{1}\left(l_{0}\right) \dot{\psi}_{2}\left(l_{0}\right)}{\dot{\psi}_{2}\left(l_{0}\right)}
$$

where $l_{0}$ is the (unique) solution of the equation $\psi_{1}(l) \psi_{2}(l)=\frac{\delta^{2}}{E^{2}}$.

Proof. The (unique) solution $\lambda_{0}$ of equation $\lambda \varphi(\lambda)=\left(\frac{\delta}{E}\right)^{2}$ in (2.10) can be obtained by the following two steps:

(a) Determine $l_{0}$ from the equation $\psi_{1}(l) \psi_{2}(l)=\frac{\delta^{2}}{E^{2}}$.

(b) Compute $\lambda_{0}=\psi_{1}\left(l_{0}\right)$.

From (5.7), (a) and (b) we conclude that

$$
\varphi\left(\lambda_{0}\right)=\psi_{2}\left(l_{0}\right) \quad \text { and } \quad \varphi^{\prime}\left(\lambda_{0}\right)=\frac{\dot{\psi}_{2}\left(l_{0}\right)}{\dot{\psi}_{1}\left(l_{0}\right)} .
$$

Consequently, (5.19) follows from $(2.10),(\mathrm{a}),(\mathrm{b})$ and $(5.20)$

Theorem 5.4. Consider the operator equation (3.2) and suppose that its unknown solution $u(x, t)$ belongs to the set $M_{p, E}$ given by (1.5). If $(3.12)$ and $\frac{\delta^{2}}{E^{2}} \leq \frac{l_{1}^{-p}}{\cosh ^{2}(\sqrt{1} T)}$ hold, then the method of generalized singular value decomposition (5.18) is optimal on $M_{p, E}$ provided the regularization parameter $\alpha$ is chosen optimally by

$$
\alpha_{0}=\frac{p+\sqrt{l_{0}} T \tanh \left(\sqrt{l_{0}} T\right)}{\cosh ^{2}\left(\sqrt{l_{0}} t\right)\left\{p+\sqrt{l_{0}} T \tanh \left(\sqrt{l_{0}} T\right)-\sqrt{l_{0}} t \tanh \left(\sqrt{l_{0}} t\right)\right\}}
$$

where $l_{0}$ is the (unique) solution of equation (5.13). In the case $t=T$ and $p>0$ there holds

$$
\alpha_{0}=\frac{T}{p}\left[\frac{1}{T} \ln \frac{E}{\delta}\right]^{2 p+1}\left(\frac{\delta}{E}\right)^{2}(1+o(1)) \quad \text { for } \delta \rightarrow 0
$$

and in the case $0 \leq t<T$ and $p \geq 0$ there holds

$$
\alpha_{0}=\frac{4 T}{T-t}\left[\frac{1}{T} \ln \frac{E}{\delta}\right]^{2 p \frac{\delta}{T}}\left(\frac{\delta}{2 E}\right)^{2 \frac{1}{T}}(1+o(1)) \quad \text { for } \delta \rightarrow 0 .
$$

Furthermore, there holds the optimal error estimate $\left\|u_{\alpha_{0}}^{\delta}(x, t)-u(x, t)\right\| \leq \omega(\delta, t)$ where $\omega(\delta, t)$ is given by $(3.18)-(3.20)$, respectively.

Proof. The result of Theorem 5.4 follows from Theorems 2.4 and 3.6 where it remains to show that $(5.21)-(5.23)$ hold. The representation $(5.21)$ follows from $(2.10)$ and Proposition 5.3 together with (3.9) and (3.10). In the case $t=T$ and $p>0$ we use that $l_{0} \rightarrow \infty$ and $\tanh \left(\sqrt{l_{0}} T\right) \rightarrow 1$ for $\delta \rightarrow 0$ and obtain from (5.21) that

$$
\alpha_{0}=\frac{\sqrt{l_{0}} T}{p \cosh ^{2}\left(\sqrt{l_{0}} T\right)}(1+o(1)) \quad \text { for } \delta \rightarrow 0 \text {. }
$$


From (5.13) we have $\frac{1}{\cosh ^{2}\left(\sqrt{T_{0}} T\right)}=l^{p} \frac{\delta^{2}}{E^{2}}$, consequently,

$$
\alpha_{0}=\frac{T}{p} l_{0}^{p+\frac{1}{2}}\left(\frac{\delta}{E}\right)^{2}(1+o(1)) \quad \text { for } \delta \rightarrow 0 .
$$

Now we use (5.17) and obtain (5.22). Finally we consider the case $0<t<T$ and $p \geq 0$. For $\delta \rightarrow 0$ we have $l_{0} \rightarrow \infty, \tanh \left(\sqrt{l_{0}} t\right) \rightarrow 1$ and $\tanh \left(\sqrt{l_{0}} T\right) \rightarrow 1$, consequently (5.21) attains the form

$$
\alpha_{0}=\frac{T}{(T-t) \cosh ^{2}\left(\sqrt{l_{0}} t\right)}(1+o(1)) \text {. }
$$

From (5.13) we obtain $e^{-\sqrt{T_{0} T}}=l_{0}^{\frac{R}{2}}\left(\frac{\delta}{2 E}\right)(1+o(1))$ for $\delta \rightarrow 0$, consequently,

$$
\begin{aligned}
\frac{1}{\cosh ^{2}\left(\sqrt{l_{0}} t\right)} & =4 e^{-2 \sqrt{t_{0}} t}(1+o(1)) \\
& =4 l_{0}^{p \frac{t}{T}}\left(\frac{\delta}{2 E}\right)^{2 \frac{t}{T}}(1+o(1)) \text { for } \delta \rightarrow 0 .
\end{aligned}
$$

Now we use (5.24), (5.25) and (5.17) and obtain (5.23)

\section{Optimal regularization methods for problem (P2)}

For problem (P2) we obtain together with (4.3) and (4.4) that the method of generalized Tikhonov regularization (2.5) consists in the determination of a regularized approximation $u_{\alpha}^{\delta}=u_{\alpha}^{\delta}(x, t)$ by solving the minimization problem

$$
\min _{u \in H} J_{\alpha}(u), \quad J_{\alpha}(u)=\left\|\frac{\sqrt{L}}{\sinh (\sqrt{L} t)} u-u_{t}^{\delta}(x, 0)\right\|^{2}+\alpha\left\|L^{\frac{2}{2}} \frac{\sinh (\sqrt{L} T)}{\sinh (\sqrt{L} t)} u\right\|^{2},
$$

hence $u_{\alpha}^{\delta}=u_{\alpha}^{\delta}(x, t)$ is the solution of the Euler equation

$$
\left(\frac{L}{\sinh ^{2}(\sqrt{L} t)}+\alpha L^{p} \frac{\sinh ^{2}(\sqrt{L} T)}{\sinh ^{2}(\sqrt{L} t)}\right) u_{\alpha}^{\delta}(x, t)=\frac{\sqrt{L}}{\sinh (\sqrt{L} t)} u_{t}^{\delta}(x, 0)
$$

of the functional $J_{\alpha}$, or equivalently, $u_{\alpha}^{\delta}=u_{\alpha}^{\delta}(x, t)$ is the solution of the symmetric, positive definite operator equation

$$
(A(t)+\alpha B(t)) u_{\alpha}^{\delta}(x, t)=u_{t}^{\delta}(x, 0)
$$

with $A(t)$ given by (4.3) and $B(t)$ given by $B(t)=L^{p-\frac{1}{2}} \frac{\sinh ^{2}(\sqrt{L} T)}{\sinh (\sqrt{L} t)}$. A further equivalent representation of $(6.2)$ is given by

$$
A(t) u_{\alpha}^{\delta}(x, t)=(I+\alpha C)^{-1} u_{\imath}^{\delta}(x, 0)
$$

with $C=L^{p-1} \sinh ^{2}(\sqrt{L} T)$ which can be interpreted as a special data smoothing method (see (5.4) for a corresponding discussion for problem (P1)).

The numerical computation of $u_{\alpha}^{\delta}(x, t)$ can effectively be done by using gradient type methods for minimizing Tikhonov's functional $J_{\alpha}$ (see Section 5 for a corresponding discussion concerning problem (P1)).

In order to prove optimality results for method (6.1) we apply Theorems 2.3 and 3.6 as well as Proposition 5.1, proceed according to the proof of Theorem 5.2 and obtain 
Theorem 6.1. Consider the operator equation (4.2) and suppose that its unknown solution $u(x, t)$ belongs to the set $M_{p, E}$ given by (1.5). If (4.9) and $\frac{\delta^{2}}{E^{2}} \leq \frac{l_{2}^{1-p}}{\sinh ^{2}\left(\sqrt{l_{1}} T\right)}$ hold, then the method of generalized Tikhonov regularization (6.1) is optimal on $M_{p, E}$ provided the regularization parameter $\alpha$ is chosen optimally by

$$
\alpha_{0}=\frac{\sqrt{l_{0}} t \operatorname{coth}\left(\sqrt{l_{0}} t\right)-1}{p+\sqrt{l_{0}} T \operatorname{coth}\left(\sqrt{l_{0}} T\right)-\sqrt{l_{0}} t \operatorname{coth}\left(\sqrt{l_{0}} t\right)}\left(\frac{\delta}{E}\right)^{2}
$$

where $l_{0}$ is the (unique) solution of the equation

$$
\frac{l^{1-p}}{\sinh ^{2}(\sqrt{l} T)}=\frac{\delta^{2}}{E^{2}}
$$

In the case $t=T$ and $p>0$ there holds

$$
\alpha_{0}=\frac{1}{p}\left[\ln \frac{E}{\delta}\right]\left(\frac{\delta}{E}\right)^{2}(1+o(1)) \quad \text { for } \delta \rightarrow 0
$$

and in the case $0 \leq t<T$ and $p \geq 0$ there holds

$$
\alpha_{0}=\frac{t}{T-t}\left(\frac{\delta}{E}\right)^{2}(1+o(1)) \quad \text { for } \delta \rightarrow 0 .
$$

Furthermore, there holds the optimal error estimate $\left\|u_{\alpha_{0}}^{\delta}(x, t)-u(x, t)\right\| \leq \omega(\delta, t)$ where $\omega(\delta, t)$ is given by $(4.14)-(4.16)$, respectively.

In the second part of this section we search for optimality results of the method of generalized singular value decomposition applied to problem (P2). For this problem we obtain with (4.3) that the method of generalized singular value decomposition (2.9) consists in the determination of a regularized approximation $u_{\alpha}^{\delta}=u_{\alpha}^{\delta}(x, t)$ according to

$$
\begin{aligned}
u_{\alpha}^{\delta}(x, t) & =\sum_{s_{i} \geq \sqrt{\alpha}} \frac{\left(u_{t}^{\delta}(x, 0), u_{i}\right)}{s_{i}} u_{i}+\frac{1}{\alpha} \sum_{s_{i}<\sqrt{\alpha}} s_{i}\left(u_{i}^{\delta}(x, 0), u_{i}\right) u_{i} \\
& =\sum_{s_{i} \geq \sqrt{\alpha}} \frac{\left(u_{t}^{\delta}(x, 0), u_{i}\right)}{s_{i}} u_{i}+\frac{1}{\alpha}\left\{A(t) u_{t}^{\delta}(x, 0)-\sum_{s_{i} \geq \sqrt{\alpha}} s_{i}\left(u_{t}^{\delta}(x, 0), u_{i}\right) u_{i}\right\}
\end{aligned}
$$

with $s_{i}=\frac{\sqrt{T_{i}}}{\sinh (\sqrt{i} t)}$ and $A(t)=\frac{\sqrt{L}}{\sinh (\sqrt{L} t)}$. The element $w(x)=A\left(t_{0}\right) u_{\imath}^{\delta}(x, 0)$ can be obtained by $w(x)=v_{t}(x, 0)$ where $v(x, t)$ is the solution of the problem

$$
\left.\begin{array}{rlrl}
v_{t t}-L v & =0 & & \text { for } t \in\left(0, t_{0}\right) \text { and } x \in \Omega \subset \mathbb{R}^{n} \\
v(x, 0) & =0 & & \text { for } x \in \Omega \\
v\left(x, t_{0}\right) & =u_{t}^{\delta}(x, 0) & & \text { for } x \in \Omega .
\end{array}\right\}
$$

Method (6.8) appears to be very cheap concerning the computational amount of work provided the eigenvalues and eigenelements of the operator $L$ are known analytically. Note that in the second representation of (6.8) only 'finite' sums occur.

In order to prove optimality results for method (6.8) we apply Theorems 2.4 and 4.6 as well as Proposition 5.3, proceed according to the proof of Theorem 5.4 and obtain 
Theorem 6.2. Consider the operator equation (4.2) and suppose that its unknown solution $u(x, t)$ belongs to the set $M_{p, E}$ given by (1.5). If (4.9) and $\frac{\delta^{2}}{E^{2}} \leq \frac{l_{1}^{1-p}}{\sinh ^{2}(\sqrt{1} T)}$ hold, then the method of generalized singular value decomposition (6.8) is optimal on $M_{p, E}$ provided the regularization parameter $\alpha$ is chosen optimally by

$$
\alpha_{0}=\frac{l_{0}\left\{(p-1)+\sqrt{l_{0}} T \operatorname{coth}\left(\sqrt{l_{0}} T\right)\right\}}{\sinh ^{2}\left(\sqrt{l_{0}} t\right)\left\{p+\sqrt{l_{0}} T \operatorname{coth}\left(\sqrt{l_{0}} T\right)-\sqrt{l_{0}} t \operatorname{coth}\left(\sqrt{l_{0}} t\right)\right\}}
$$

where $l_{0}$ is the (unique) solution of equation (6.5). In the case $t=T$ and $p>0$ there holds

$$
\alpha_{0}=\frac{T}{p}\left[\frac{1}{T} \ln \frac{E}{\delta}\right]^{2 p+1}\left(\frac{\delta}{E}\right)^{2}(1+o(1)) \quad \text { for } \delta \rightarrow 0
$$

and in the case $0 \leq t<T$ and $p \geq 0$ there holds

$$
\alpha_{0}=\frac{4 T}{T-t}\left[\frac{1}{T} \ln \frac{E}{\delta}\right]^{2+2(p-1) \frac{t}{T}}\left(\frac{\delta}{2 E}\right)^{2 \frac{1}{T}}(1+o(1)) \quad \text { for } \delta \rightarrow 0 .
$$

Furthermore, there holds the optimal error estimate $\left\|u_{\alpha_{0}}^{\delta}(x, t)-u(x, t)\right\| \leq \omega(\delta, t)$ where $\omega(\delta, t)$ is given by $(4.14)-(4.16)$, respectively.

\section{References}

[1] Baumeister, J.: Stable Solution of Inverse Problems. Braunschweig: Vieweg 1987.

[2] Engl, H. W.: Regularization methods for the stable solution of inverse problems. Surv. Math. Ind. 3 (1993), 71 - 143.

[3] Groetsch, C. W.: The Theory of Tikhonov Regularization for Fredholm Equations of the First Kind. Boston: Pitman 1984.

[4] Hackbusch, W.: Theorie und Numerik elliptischer Differentialgleichungen. Stuttgart: Teubner-Verlag 1986.

[5] Hadamard, J.: Lectures on Cauchy's Problem in Linear Partial Differential Equations. New Haven: Yale University Press 1923.

[6] Hanke, M. and P. C. Hansen: Regularization methods for large-scale problems. Surv. Math. Ind. 3 (1993), $253-315$.

[7] Hao, D. N.: A mollification method for ill-posed problems. Numer. Math. 68 (1994), 469 -506 .

[8] Hofmann, B.: Regularization for Applied Inverse and Ill-Posed Problems (Teubner-Texte zur Mathematik: Vol. 85). Leipzig: B. G. Teubner Verlagsges. 1986.

[9] Ivanov, V. K., Vasin, V. V. and V. P. Tanana: Theory of Linear Ill-Posed Problems (in Russian). Moscow: Nauka 1978.

[10] Krein, S. and Y. I. Petunin: Scales of Banach spaces. Russian Math. Surveys 21 (1966), $85-159$.

[11] Lavrentiev, M. M.: Some Improperly Posed Problems in Mathematical Physics. Berlin: Springer-Verlag 1967. 
[12] Louis, A. K.: Inverse und schlecht gestellte Probleme. Stuttgart: Teubner-Verlag 1989.

[13] Mair, B. A.: Tikhonov regularization for finitely and infinitely smoothing operators. SIAM J. Math. Anal. 25 (1994), $135-147$.

(14) Schröter, T. and U. Tautenhahn: On the optimality of regularization methods for solving linear ill-posed problems. Z. Anal. Anw.. 13 (1994), $697-710$.

[15] Schröter, T. and U. Tautenhahn: On optimal regularization methods for the backward heat equation. Z. Anal. Anw. 15 (1996), $475-493$.

[16] Seidman, T. I.: 'Optimal Filtering' for some ill-posed problems. In: Wave Propagation and Inversion (eds.: W. Fitzgibbon and M. Wheeler). Philadelphia: SIAM 1992, pp. 108 -123 .

[17] Tautenhahn, U.: Optimality for linear ill-posed problems under general source conditions. Preprint IP 5/1996. Techn. Univ. Chemnitz-Zwickau (submitted).

[18] Tikhonov, A. N. and V. Y. Arsenin: Solution of Ill-Posed Problems. New York: Wiley 1977.

[19] Vainikko, G. M.: On the optimality of methods for ill-posed problems. Z. Anal. Anw. 6 (1987), $351-362$.

[20] Vainikko, G. M. and A. Y. Veretennikov: Iteration Procedures in Ill-Posed Problems (in Russian). Moscow: Nauka 1986.

Receiced 19.04.1996 